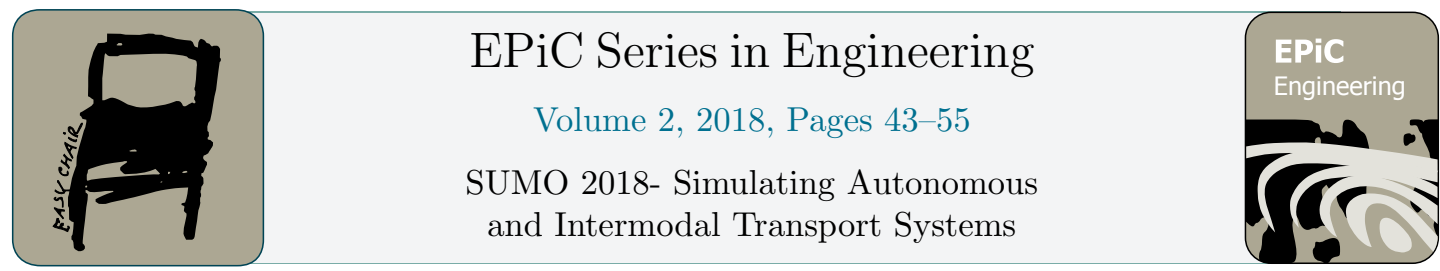

\title{
Monaco SUMO Traffic (MoST) Scenario: A 3D Mobility Scenario for Cooperative ITS
}

\author{
Lara CODECÁ ${ }^{1}$ and Jérôme HÄRRI ${ }^{1}$ \\ EURECOM - Communication Systems Department \\ 06904 Sophia-Antipolis, France \\ [codeca, haerri] @eurecom.fr
}

\begin{abstract}
Cooperative Intelligent Transportation Systems (C-ITS) are a viable solution when it comes to the optimization of the ever-growing population moving in the cities. C-ITS studies have to deal with telecommunications issues and location errors due to the urban environment, while keeping into account realistic mobility patterns. A detailed and state of the art scenario is complex to generate and validate. There is a trade-off between precision and scalability. Additionally, precise information may be problematic to obtain or use due to privacy issues. There are some general-purpose freely-available scenarios, but none of them provides a 3D environment with intermodal traffic. Nonetheless, the 3D environment is a requirement to have reliable C-ITS simulations in a realistic setting, and the importance of intermodal mobility cannot be overlooked in planning the future of smart cities. The Monaco SUMO Traffic (MoST) Scenario aims to provide a state of the art 3D playground with various kind of vehicles, vulnerable road users and public transports to test C-ITS solutions. This paper presents the data requirements, characteristics, possible use cases, and finally, the limitations of MoST Scenario.
\end{abstract}

Keywords 3D Traffic Scenario, Intermodal Transport Planning, Cooperative Intelligent Transportation Systems

\section{Introduction}

Our cities are complex systems in constant evolution. The information that is collected from various sources is enhancing our view and understanding of their dynamics. C-ITS are using mobility information from vehicles and vulnerable road users to improve traffic and the quality of life in the cities and their surroundings. This objective requires understanding not only of traffic flows theory and telecommunications, but also human behavior and social interactions.

We are always looking for methods and tools to test theories and develop state of the art C-ITS protocols and applications, improving road traffic, safety, and security. Mobility patterns influence telecommunications in a critical way and they must be evaluated together to provide realistic and robust solutions. A simulation environment capable of providing an interactive smart city scenario has limitations and requirements coming from both mobility and 
telecommunication simulators, and their possible interaction. In literature we can find surveys such as [1] and [2] that are able to provide a good overview on the different mobility and network simulators available to the scientific community, with their pros and cons, depending on the task at hand.

To appeal at the largest audience, we chose Simulator of Urban MObiltiy (SUMO) [3] as mobility simulator because its community is very active on Intelligent Transportation Systems (ITS) topics and it provides a socket interface that allows easy communication with other simulators. For example, OMNet++ and NS3 are network simulators widely used in the Vehicular Ad Hoc Network (VANET) community, and they both can be interfaced with SUMO using respectively Vehicles in Network Simulation (VEINS) [4] and iTETRIS [5].

\section{$1.1 \quad$ C-ITS Requirements}

An interactive mobility scenario for C-ITS has to provide a complete playground that integrates different aspects. We need a multidimensional environment to work on geo-location and telecommunication aspects. It has to be highly populated, with congested traffic patterns to work on alternative and intermodal mobility optimizations. Last but not least, it has to be scalable and controllable.

An environment able to provide all the above-mentioned features is the Principality of Monaco. The city itself covers an area of $2 \mathrm{~km}^{2}$ and the greater area (composed of French cities adjacent to Monaco) is about $70 \mathrm{~km}^{2}$. The size of the city provides a small and more controllable environment, reasonable to model with a microscopic mobility simulator.

Some of the reasons why this kind of interactive mobility playground is important, are available at [6], but here we will discuss them further, explaining how the MoST Scenario is implemented and can be used.

Mobility Traffic patterns in Monaco follow the typical behavior seen in many European capitals. Most of the people that work in the city are commuters and they live in the greater area, for both economical and logistic reasons. In the morning, there is a directional traffic congestion converging to city, and during the evening, the city experience a congested traffic outflow. Monaco is a well-organized city. In order to accommodate all the commuters, it provides many parking areas with a fairly developed public transports network. Nonetheless, the infrastructures in place are not able to alleviate traffic congestion during rush hours. Hence, Monaco city presents a perfect playground to study city-scale advanced parking management solutions, intermodal and alternative transport modes applications.

Territory Monaco is built on the mountains' slope, facing the Mediterranean sea. Like many coastal cities close to a mountain region, it has a layered topology full of tunnels and bridges. These features present a multidimensional environment more realistic compared to a flat surface. This kind of topology allows the study of precise positioning and three-dimensional propagation models, and their impact on C-ITS. Additionally, these multidimensional features, in conjunction with realistic emission models, can be useful in studying eco-routing based on different factors, such as energy consumption and/or environmental impact.

Pedestrians Tourism in Monaco is an important source of revenue. On top of the pedestrian commuters that are headed to work, there are waves of tourists that reach the city mainly by tour buses and public transports. A large scale mobility scenario able to integrate vulnerable users, can be locally coupled with specific crowds and multi-agents simulators (e.g.: Menge 
Crowd Simulator [7]), that given their complexity, would not be otherwise scalable over an entire city.

In the following sections, we are going to provide detailed information on the MoST Scenario, its features, possible use cases, and the road ahead, with the future work. The MoST Scenario is freely available to the community under GPLv3 license. It can be downloaded from https://github.com/lcodeca/MoSTScenario.

\section{Related Works}

Discussing the state of the art on the mobility scenarios, we decided to focus only on the one available for SUMO.

Cologne One of the most cited mobility scenario for sumo is the TAPAS Cologne [8] Scenario, which includes road networks imported from OpenStreetMap (OSM) and two possible traffic demands: one from 6:00 to 8:00 in the morning, and the other over 24 hours. Unfortunately, this scenario is cumbersome and requires additional work to improve the network quality, to verify how routes are mapped onto both the network and the traffic demand. Additionally, the buildings definition is not meant for a network simulator, making it not directly usable in C-ITS simulations.

Bologna A realistic traffic scenario from the city of Bologna [9, 10], and built in the iTETRIS [5] framework, provides a very good starting point for the community, but it has some limitations. The traffic demand is only defined over one hour, and the size of the scenario, which is relatively small, provides only one neighborhood. In this setting, the elevation does not matter, given that the modeled area is flat.

Austria On a completely different scale, the ITS Austria West scenario [11] presents a real life traffic monitoring system that uses a mesoscopic version of SUMO [12]. This scenario is a very good example of the effort required to build and maintain a large scale traffic scenario. The project monitors an infrastructure with around 245,000 nodes and 320,000 edges with a total length of $27,000 \mathrm{~km}$. They have five different traffic information sources providing floating car data and a traffic demand model for the simulation of 1.2 million routes and 1.6 million vehicles. Unfortunately this scenario is not freely available to the community as it relies on proprietary information. Nonetheless, it is a very good example of the performances capabilities of SUMO.

Luxembourg From the country of Luxembourg two possible scenarios are available. VehiLux [13] is a general purpose scenario that presents the same issues as TAPAS Cologne. Additionally, the scenario has been discontinued. More recently, the Luxembourg SUMO Traffic (LuST) Scenario [14] has been created with the focus on C-ITS and telecommunications. The LuST Scenario is based on the same tools and sources of information used by VehiLux, but it has been fine tuned and hand-checked in order to provide realistic and reliable data. And finally, the mobility has been enhanced and validated. Although this scenario is widely used in VANET simulations, it does not provide any elevation information and the multimodal capabilities are only partial, focusing only on vehicles and not pedestrians. 
At the best of our knowledge, MoST Scenario is the first freely-available mobility scenario with elevation information for streets and buildings, and fully functional intermodal mobility for pedestrian.

\section{Monaco SUMO Traffic Scenario Features}

The initial dataset is extracted from OSM and it provides many of the data required to build the scenario. OSM is a collection of crowd-sourced information and the accuracy is not always consistent [15]. A more detailed version of the geographical information is extracted from the Institut Géographique National $(\mathrm{IGN})^{1}$ BD TOPO $^{\circledR}$ database under the ISNPIRE licence ${ }^{2}$. Here we can find detailed data concerning altitude and the land registry. This information is integrated with the street topology and the Points Of Interest (POIs) dataset to obtain a reliable multidimensional model of the city.

This initial dataset is then integrated and refined using data provided by public websites from the Monaco and France. Public transports are gathered from OSM, Compagnie des Autobus de Monaco (CAM) ${ }^{3}$, and Provence-Alpes-Côte d'Azur (PACA) Mobilité ${ }^{4}$, where it can be found location and schedule for buses and trains, and the location of the electric bicycle stations with their availability.

In the data extracted from OSM we find the location of parking lots, train stations, bus stops, taxi stands, and bicycle sharing points; these POIs will be used to build the main infrastructure for multimodality and public transportation. The location of additional POIs (e.g.: museums, tourist attractions and parks, shops and restaurants, offices and facilities, and schools) is used to improve the realism of the activity-based mobility we are going to generate. The Monaco Parking website ${ }^{5}$ provides location, capacity, schedule, prices and availability for many of the parking lots in the city.

\begin{tabular}{cc}
\hline Total Area & $73.64 \mathrm{~km}^{2}$ \\
\hline Edges length & $587,452.37 \mathrm{~m}$ \\
Edges with 1 lane & 653 \\
Edges with 2 lanes & 2,935 \\
Edges with 3 lanes & 661 \\
\hline Total Junctions & 4,667 \\
Priority & 487 \\
Traffic Light & 831 \\
Roundabouts & 24 \\
\hline
\end{tabular}

\begin{tabular}{ccc}
\hline Motorway & $16,817.64 \mathrm{~m}$ & $(5,458.85 \mathrm{~m})$ \\
Primary & $44,824.26 \mathrm{~m}$ & $(4,665.59 \mathrm{~m})$ \\
Secondary & $90,782.24 \mathrm{~m}$ & $(5,778.83 \mathrm{~m})$ \\
Tertiary & $67,571.56 \mathrm{~m}$ & $(2,787.15 \mathrm{~m})$ \\
Service & $3,789.67 \mathrm{~m}$ & \\
Residential & $278,468.31 \mathrm{~m}$ & \\
\hline Pedestrian & $21,867.88 \mathrm{~m}$ & \\
Steps & $17,946.28 \mathrm{~m}$ & \\
\hline Railway & $26,694.11 \mathrm{~m}$ & \\
\hline
\end{tabular}

Table 1: MoST Scenario network topology.

\subsection{Network Topology}

The above-mentioned information have been processed and merged in an OSM-like file in order to be handled with tools such as JOSM [16] and netconvert [17]. In Table 1 we present a summary of the static network information. The MoST Scenario covers an area of approximatively

\footnotetext{
${ }^{1}$ https://geoservices.ign.fr

${ }^{2}$ Since May 9, 2011, the INSPIRE directive requires the release of data for research purposes, in accordance with the requirements of the CE Regulation n 976/2009

3 http://cam.mc

${ }^{4}$ https://www.pacamobilite.fr

${ }^{5}$ https://monaco-parking.mc
} 
$73 \mathrm{~km}^{2}$, it includes all the road types from motorway to residential, and model both pedestrian paths and stairways (steps).

Intersections In order to have a working SUMO simulation, the intersections were checked manually for correctness using an iterative process with JOSM, netconvert and SUMO to ensure that they did not represent an unrealistic bottleneck for the traffic flows. Additionally, the default size for roads and sidewalks had to be reduced in order to be closer to reality. Concerning the tunnels, we introduced new categories in OSM in order to tag them and import them in SUMO. Even if this information is not important for SUMO, it is very important for the telecommunication simulator.

In the Principality of Monaco the vast majority of intersections is priority based, with a limited number of Traffic Light Systems (TLS) in place. Nonetheless, in the scenario it has been necessary to regulate most of the intersections with a TLS to simulate a more realistic interaction between pedestrian and vehicles. In SUMO, vehicles always stop to let the pedestrians waiting at the zebra crossroad to pass, creating unrealistic congestion when it comes to dense scenarios. In reality, there will be a mutual understanding between vehicles and pedestrians, and their crossing will happen in waves. In simulation, this behavior is better modeled with a TLS regulated intersection.

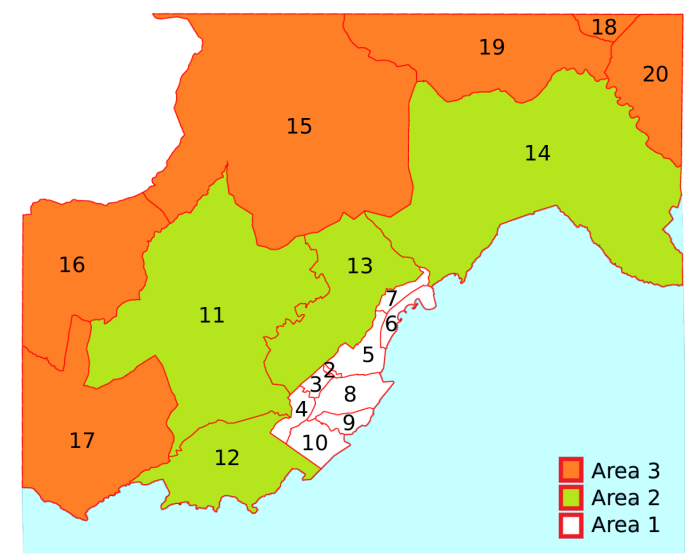

Figure 1: Traffic Assignment Zones (TAZ) colored by areas.

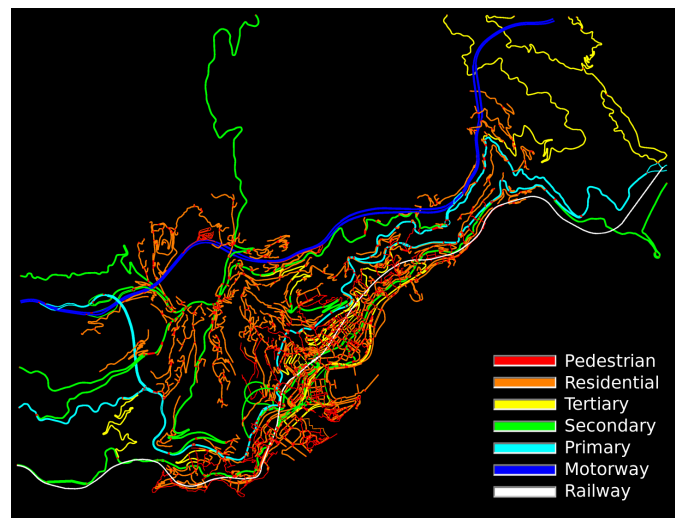

Figure 2: MoST Scenario road topology colored by priority.

Areas The MoST Scenario is divided in various Traffic Assignment Zone (TAZ) ${ }^{6}$, and they coincide with the administrative boundaries of the territory in question. In Figure 1 all the TAZ are numbered and they are colored by logical areas (from 1 to 3 ). The reference for the TAZ enumeration is in Table 2. Area 1 is the Principality of Monaco, Area 2 is composed by the French villages on the border, and Area 3 is composed by the French villages further away. Figure 2 shows the topology of MoST Scenario with the streets colored by priority. For example, the motorway (in blue) runs in the outskirts of the urban area, and the railway (in white) goes through all the villages, following the coastline.

\footnotetext{
${ }^{6}$ In this context, a traffic assignment zone is a geographical area used to model the traffic patterns and the traffic demand.
} 
Based on Figures 1 and 2 we can discuss and understand the topology in detail. In Area 1 we find the Principality of Monaco, with all its neighborhoods. In Table 2 it is possible to find the number of Points of Interest (PoIs) extracted from the datasets associated with each TAZ and the area it covers. In Area 2 we find the French villages on the border with Monaco. Areas 1 and 2 are the most detailed and close to reality. Area 3 is fundamental to model the traffic patterns in in the area, but its level of detail is limited. Only the main roads are represented to allow the correct inflow (outflow) to (from) the inner areas and the proper definition of the public transports. In both Areas 1 and 2 we can find the pedestrian areas, but the stairways definition can be found mainly in Area 1.

\begin{tabular}{ccccc}
\hline Area & TAZ & TAZ Name & PoIs & Area $\left[\mathrm{m}^{2}\right]$ \\
\hline 1 & 1 & Monaco & 21,150 & $3,150,797$ \\
\hline 1 & 2 & Sainte Devote & 296 & 29,696 \\
1 & 3 & Les Monegetti & 1,482 & 137,458 \\
1 & 4 & Jardin Exotique & 2,492 & 304,780 \\
1 & 5 & Monte Carlo & 5,303 & 568,848 \\
1 & 6 & Larvotto & 1,243 & 385,032 \\
1 & 7 & La Rousse & 2,968 & 263,333 \\
1 & 8 & La Condamine & 2,072 & 589,846 \\
1 & 9 & Monaco Ville & 1,627 & 242,837 \\
1 & 10 & Fontvieille & 2,302 & 438,647 \\
\hline 2 & 11 & La Turbie & 36,039 & $10,457,135$ \\
2 & 12 & Cap d'Ail & 15,831 & $2,905,470$ \\
2 & 13 & Beausoleil & 27,372 & $3,711,430$ \\
2 & 14 & Roquebrune & 51,991 & $13,046,118$ \\
\hline 3 & 15 & Peille & 6,158 & $12,554,684$ \\
3 & 16 & La Trinite & 3,734 & $4,229,112$ \\
3 & 17 & Eze & 12,839 & $5,388,225$ \\
3 & 18 & Sainte Agnes & 605 & 278,627 \\
3 & 19 & Gorbio & 5,576 & $4,215,247$ \\
3 & 20 & Menton & 4,472 & $1,928,595$ \\
\hline
\end{tabular}

Table 2: TAZ definition and weights.

Public Transports Public transports and parking lots are defined for the entire scenario. This allows to model the intermodal traffic in detail, following the P+R (Park and Ride) paradigm already available in many cities. In the MoST Scenario we have 176 bus stops covered by 24 routes, and 12 train stations, covered by 2 routes. The coverage of the public transports is shown in Figure 3, in red. It is easy to notice that only the coastline is served by public transports, and this is a well-known issue in this area. In the scenario we defined 131 parking lots, and for each of them, we computed which are the three closest alternatives in case a parking lot is full.

3D Environment The multidimensional environment in which we live heavily influence daily decisions. For example, hybrid and electric vehicles are getting more common in our cities: it is 


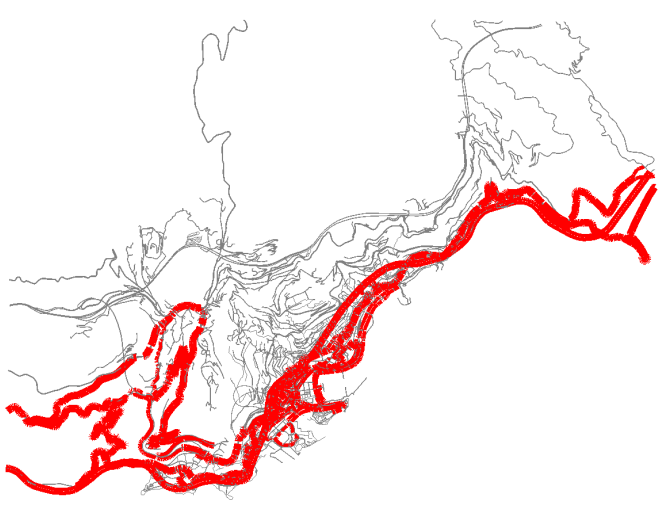

Figure 3: Public Transports coverage (in red). tion.

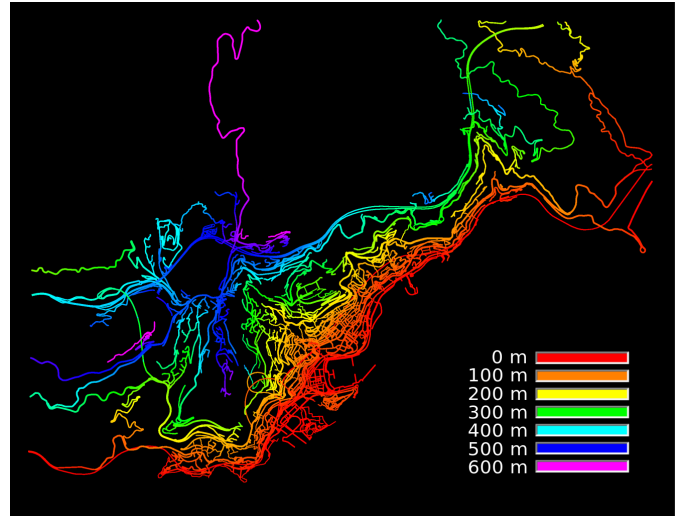

Figure 4: Network topology colored by eleva-

common knowledge that they reduce the pollution and help us save money on fuel. Nonetheless, it is complicated to find a study on the efficiency of hybrid (or electric) vehicles when it comes to city with mountain-like elevation and slopes. Additionally, when it comes to smart and interconnected cities planning, telecommunications tend to be a given. In the study of C-ITS, it is well known that elevation, slopes and obstacles pose challenging issues that are complicated to study in simulation, due to models complexity and lack of data. For these reasons, in the MoST Scenario there are about 15,000 buildings with their shape and elevation, 96 bridges and a total of 74 tunnels. The elevation map has been extracted from BD TOPO ${ }^{\circledR}$ database, and averaged out in the tunnels and where the information was missing. Figure 4 shows the elevation of the streets in the scenario. As expected, streets near the coast are at sea-level, but the slope change rapidly from 0 to 600 meters.

\subsection{Traffic Demand}

The MoST Scenario provides, by default, a mobility for the morning rush hour. The traffic patterns are characterized by inbound traffic congestion directed to the Principality of Monaco. Due to the lack of live traffic data and real traffic counters, we generated a synthetic traffic demand based on statistical information on the population, and a simplified activity-based mobility generation model [18].

We identified the TAZ as shown in Table 2, and in order to compute their absolute weight, we divided the number of PoIs $\left(4^{\text {th }}\right.$ column $)$ by the area $\left(5^{\text {th }}\right.$ column $)$ in question. The OriginDestination (OD) matrices are defined over the three areas and for each different type of transport. For example, it can be defined such as 2500 trucks from Area 3 to Area 1; hence, the real weight of a TAZ must be normalized in the pool of origin (or destination) area in question.

Figure 5 shows the traffic demand over the morning, divided by type of vehicles. This traffic demand of 20,000 entities has been generated as a normal distribution with mean around 08h15 $\mathrm{AM}$ and a standard deviation of 1 hour and 30 minutes. Figure 6 shows the summarized result of the SUMO simulation. The relative speed is computed taking into account the maximum speed allowed on the streets. The waiting time is defined as the amount of time in which the vehicles are slower than $0.1 \mathrm{~m} / \mathrm{s}$. Analyzing the graphs in Figure 6 with the running vehicles $\left(1^{\text {st }}\right.$ graph) and the mean relative speed ( $3^{\text {st }}$ graph), it is observable that the scenario is congested, but given that there are virtually no waiting vehicles ( $2^{\text {nd }}$ graph), the scenario is not saturated. 


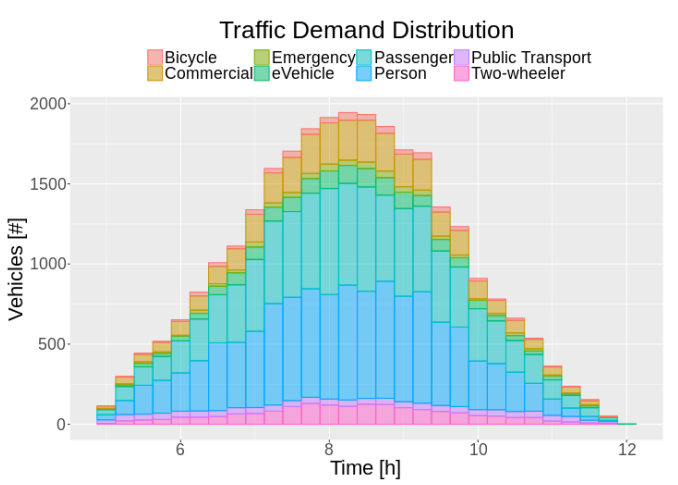

Figure 5: Traffic Demand by vTypes.

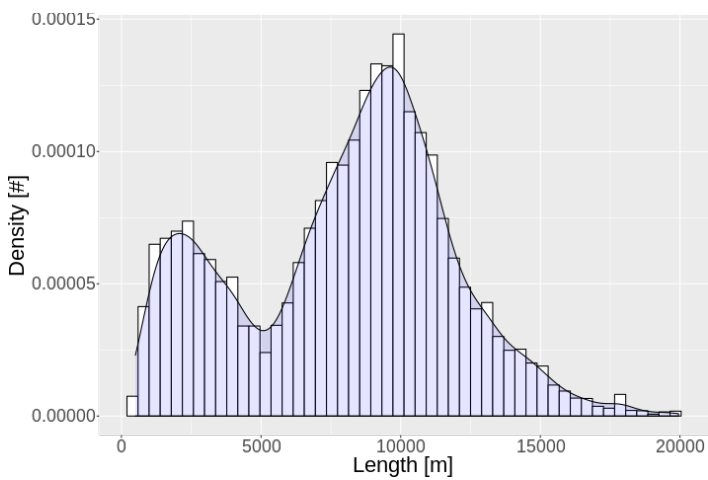

(a) Route length distribution.

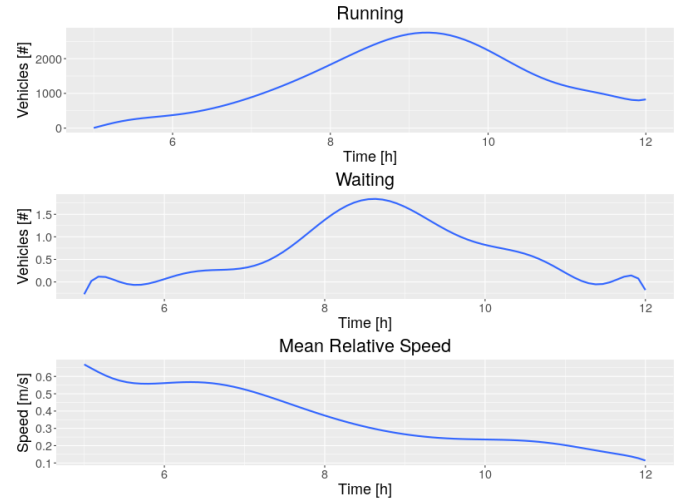

Figure 6: Simulation results summary.

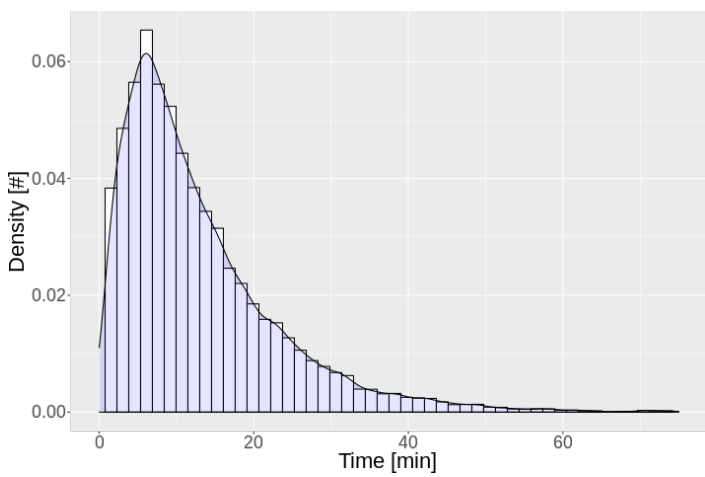

(b) Time lost distribution.

Figure 7: Route length and time loss distribution of all the vehicles in the simulation.

Figure 7 shows route length and time loss distribution of all the vehicles in the simulation. More precisely, the distribution of the route length is shown in Figure 7(a). where the two bells shaped curve represents the traffic internal to the Principality of Monaco (less than $5 \mathrm{~km}$ ) and the traffic coming from France (Area 2 and 3). The distribution of the time lost by each vehicle is presented in Figure 7(b). Here we can notice that the vast majority of the vehicles has to endure delays due to the traffic congestion that are shorter than 20 minutes.

Figure 8 shows the impact of intermodal mobility for pedestrians. More precisely, Figure 8(a) shows the route length distribution for the walking part of the trip. The majority of the pedestrian are able to reach their destination walking less than $1 \mathrm{~km}$. Note that a share of the pedestrians moving inside the city were purposefully not allowed to use public transports, to represent tourists moving around in the Principality of Monaco. The route length distribution of the part of the trip spent riding public transports is presented in Figure 8(b). Here we can notice a spike in the shorter distances (less than $2.5 \mathrm{~km}$ ), and it coincides with the public transports inside the Principality of Monaco itself. This is partially an artifact of the simulation, but reflect the lack of public transport routes away from the coastline. Finally, Figure 8 shows the distribution of the time spent at the stop waiting for the public transports. In this specific scenario, we decided to schedule the public transports with a frequency of 15 minutes to better 


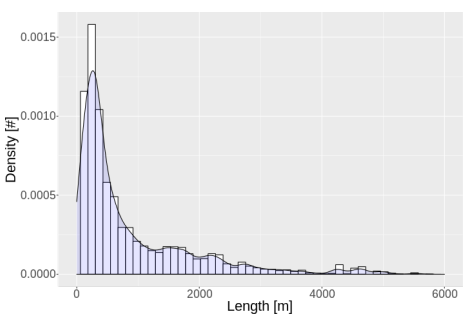

(a) Walking length distribution.

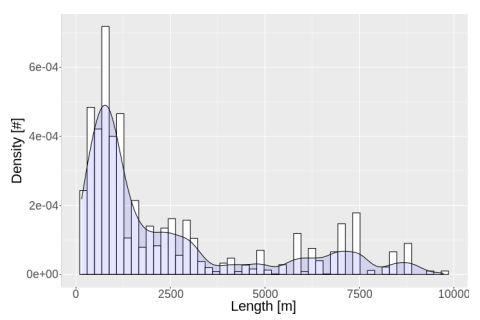

(b) Route length distribution of the public transport rides.

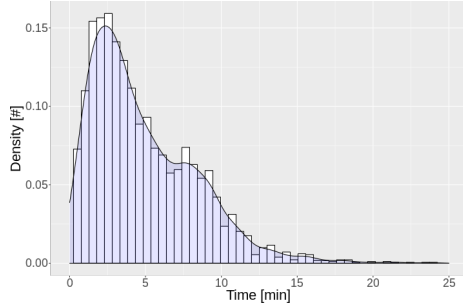

(c) Public transports waiting time distribution.

Figure 8: Route length distribution and average speed distribution of all the vehicles in the simulation.

represent the morning rush hour timetable. This decision is reflected in the distribution of the waiting time, where the vast majority of the people has to wait less than 15 minutes.

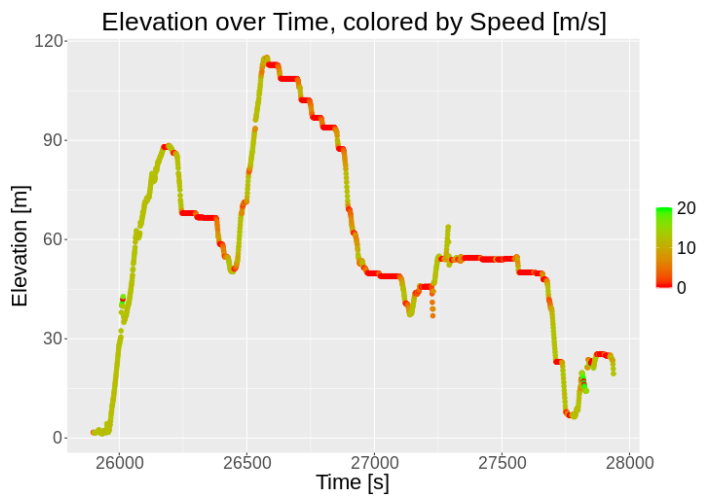

(a) Elevation.

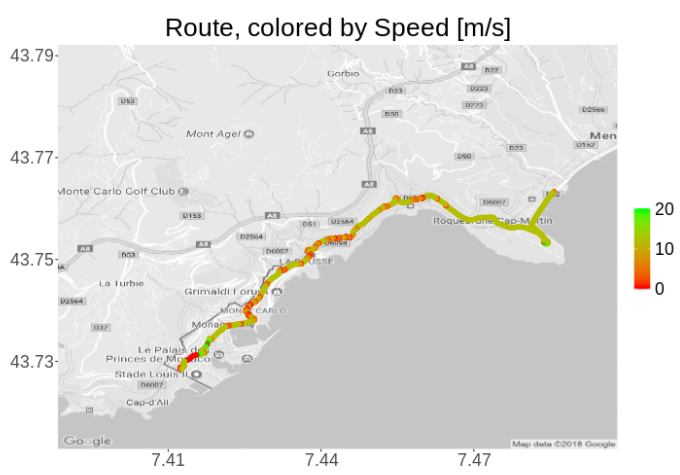

(b) Route.

Figure 9: Vehicle: Variation of elevation and speed over time.

Figure 9 shows some information on the route of a vehicle. We randomly selected a passenger vehicle to show the variation in its elevation and speed (Figure 9(a)) and its route (Figure 9(b)) as an example of a possible trip that can be found in the MoST Scenario. In this case, its elevation varies from a minimum of $1.27 \mathrm{~m}$ to a maximum of $115.0 \mathrm{~m}$, with a median of 54.10 $\mathrm{m}$. Its maximum speed reached is $25.75 \mathrm{~m} / \mathrm{s}$, with a mean of $5.11 \mathrm{~m} / \mathrm{s}$. The total route length is $10,434.68 \mathrm{~m}$ and its time lost due to traffic ${ }^{7}$ is $1260.19 \mathrm{~s}$.

We randomly selected a person to show the behavior of intermodal planning and we presented it in Figure 10. Elevation and speed patterns are shown in Figure 10(a) and the route in Figure 10(b). This is an example of a possible person trip that can be found in the MoST Scenario. In this case, its elevation varies from a minimum of $6.06 \mathrm{~m}$ to a maximum of 78.9 $\mathrm{m}$, with a median of $53.07 \mathrm{~m}$. Here is interesting to notice the variation in the speed, and it easily noticeable where the person was on a bus, thanks to the elevated speed (in green) visible in both Figures 10(a) and 10(b), and where the person was walking (in red). In this example,

\footnotetext{
${ }^{7}$ The time lost due to traffic is the timeLost computed by SUMO.
} 


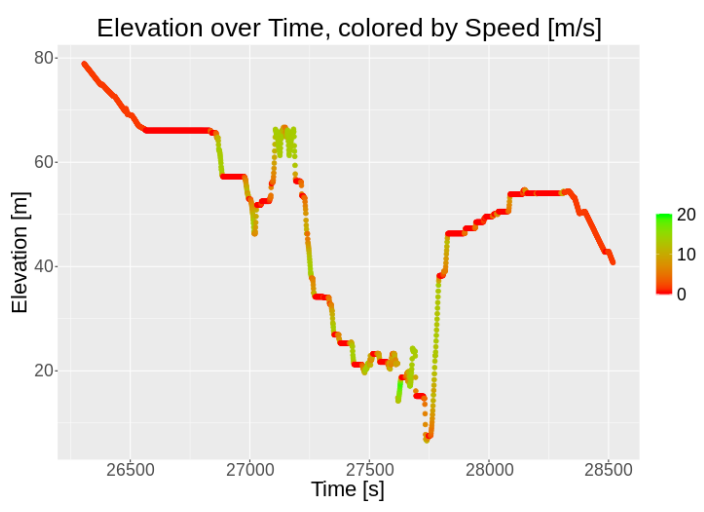

(a) Elevation.

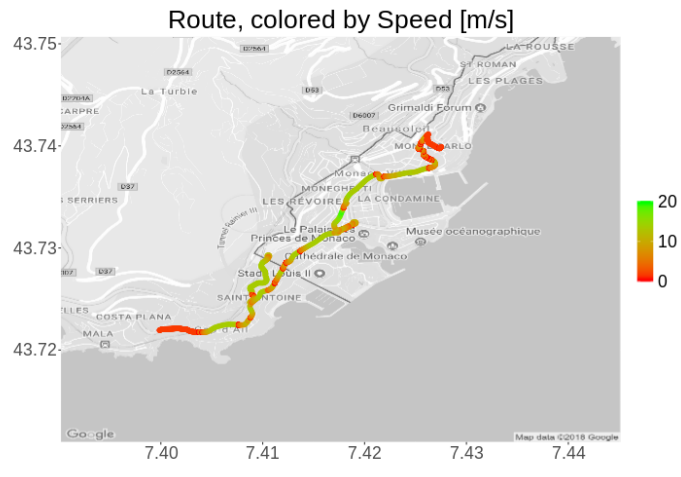

(b) Route.

Figure 10: Person: Variation of elevation and speed over time.

the person:

- walked $322.35 \mathrm{~m}$,

- waited $270 \mathrm{~s}$ for the bus,

- rode it for $56 \mathrm{~s}(334.62 \mathrm{~m})$

- waited for another bus for $87 \mathrm{~s}$

- rode it for $1339 \mathrm{~s}(4876.21 \mathrm{~m})$

- and finally walked for $240.5 \mathrm{~m}$ to reach the desired destination.

Figures 10 and 9 are meant to provide an example of what kind of trips are available in the mobility provided by MoST Scenario. In the following section, we are going to discuss a tool meant to easily generate other mobility traces.

\subsection{Mobility Generator}

Given the impossibility at the moment to validate the traffic mobility, we wanted to have and provide a tool able to easily generate various traffic demands. Starting from a population and an OD definition, it generates the SUMO trip definition for the mobility. These definitions should be validated with duarouter, a tool provided by SUMO.

A snippet of traffic demand definition is shown in Listing 1. Starting from the population size (line 2), a percentage (line 5) of it is given to each vType or vTypeDistribution, and more precisely, it is divided among the OD defined (line 6, composition). This imply that the sum of all the perc (line 11 and 17) must be 1, and for each vType, the sum of all the percentages (line 5) must be 1 . The possible options depend on the vehicle (or person) in question. It is possible to have person trips based on public transports (line 14) or just walking (line 8). Concerning the vehicles, it is possible to set the destination to a parking lot, and keep them there until the end of the simulation.

The range of vehicles (and persons) supported by this scenario is the one provided by SUMO and specified in Table 3.

Additionally, it is possible to define which percentage of the population comes from inside the area covered by the scenario, and which percentage comes from specific gateways (usually 


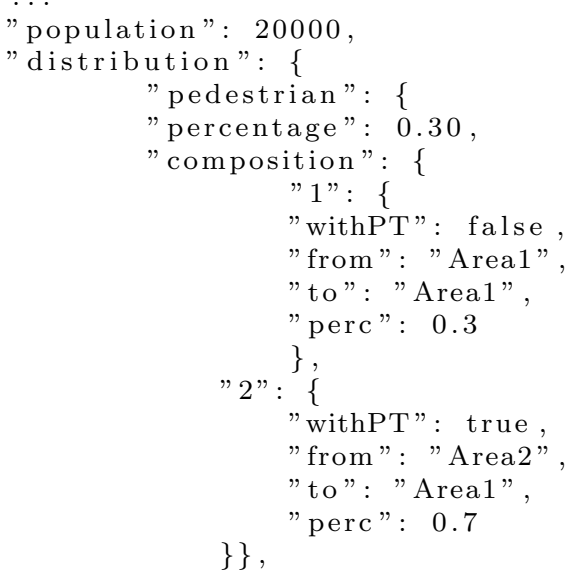

Listing 1: Simplified example of a traffic demand definition.

\begin{tabular}{cl}
\hline vClass & Description \\
\hline evehicle & $\begin{array}{l}\text { Special vehicles with } \\
\text { user-defined characteristics. }\end{array}$ \\
\hline bus & $\begin{array}{l}\text { Part of } \\
\text { public transports. }\end{array}$ \\
\hline $\begin{array}{c}\text { pedestrian } \\
\text { bicycle }\end{array}$ & $\begin{array}{l}\text { Vulnerable } \\
\text { road users. }\end{array}$ \\
\hline $\begin{array}{c}\text { motorcycle } \\
\text { moped }\end{array}$ & $\begin{array}{l}\text { In the vTypeDistribution } \\
\text { for two-wheelers. }\end{array}$ \\
\hline & $\begin{array}{l}\text { Normal vehicles } \\
\text { (defined as vTypeDistribution }\end{array}$ \\
passenger & with five different types of vehicle). \\
\hline emergency & \\
taxi & \\
coach & $\begin{array}{l}\text { In the vTypeDistribution } \\
\text { delivery } \\
\text { truck } \\
\text { trailer }\end{array}$ \\
\hline
\end{tabular}

Table 3: vType and vClasses definition.

motorways and/or external edges). Finally, the traffic demand must be defined over a time interval (defined in seconds) and the rush hour is defined specifying mean and standard deviation of a normal distribution.

\section{Use Cases and Future Work}

MoST is a general-purpose traffic scenario. Its aim is to be a simple and reliable tool to support scientific research. Our plan is to use it to work on intermodal mobility planning and optimization, eco-routing for hybrid vehicles, parking optimization for $\mathrm{P}+\mathrm{R}$ solutions and we want to explore the impact of elevation on C-ITS.

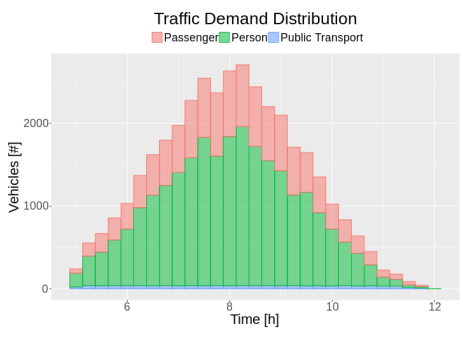

(a) Simple, only three classes.

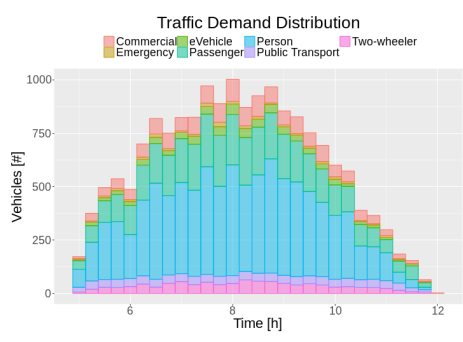

(b) Larger standard deviation.

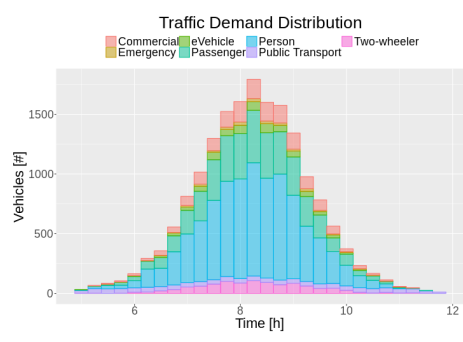

(c) Smaller standard deviation.

Figure 11: Examples of various traffic demands.

Some examples of the traffic demands that can be generated with the tool discussed in Section 3.3 are shown in Figure 11. Figure 11(a) shows a traffic demand containing only pedestrians, passenger vehicles and public transport. This kind of demands can be used to optimize the presence of public transports and to study their impact on the traffic congestion. 
Figures 11(b) and 11(c) are an example of a traffic demand with different standard deviations in the departure times. Given that the size of the population is still the same, this kind of variation in the traffic demand can be used to study the impact of varying the departure time on the traffic congestion and travel times while respecting constraints (such as the arrival time).

This 3D intermodal traffic scenario is still in active development and the future work will be driven by the community that is using it and their necessity.

The primary enhancement will focus on intermodal mobility integration. At the moment only buses and trains are considered as public transports. In the future, we want to include taxis and shared rides. Additionally, the only means of parking available at the moment are the parking lots. We would like to find a reliable source for the location of car parks along the road and integrate them in the scenario.

Another enhancement concerns the vulnerable road users and the integration of the twowheelers in the traffic. The MoST Scenario is not bicycles friendly at the moment, and it is due to lack of information. We decided to have pedestrians and cyclists share the pedestrian paths, but we would like to gather precise information to increase the realism. Additionally, we want to work on the two-wheelers model to explore this option when it comes to intermodal traffic.

Last but not least, we are actively looking for real and precise traffic information from the area in order to generate and validate a realistic mobility that reflects the Principality of Monaco and its surroundings.

\section{Conclusions}

We presented the Monaco SUMO Traffic (MoST) Scenario in details, starting with the dataset used in the generation, through the decisions made to have a scenario that works out of the box, and finally we presented a tool to generate various traffic demands starting from the one provided. The MoST Scenario provides a state of the art 3D playground with various kind of vehicles, vulnerable road users and public transports to work on Cooperative Intelligent Transportation Systems (C-ITS) solutions.

The MoST Scenario covers an area of approximatively $70 \mathrm{~km}^{2}$, that includes three logical areas and 20 Traffic Assignment Zone (TAZ). It provides the location of Points of Interest (PoIs), shape and location for the buildings, and elevation of buildings and streets. Public transports are based on buses and trains, with more than 150 stops and more than 20 routes. The scenario is provided with mobility traces that represents a morning rush hour with intermodal traffic, and a tool is available to easily modify the traffic demand to obtain the desired traffic conditions.

The MoST Scenario is the first freely-available mobility scenario with elevation information for streets and buildings, and fully functional multimodal mobility for pedestrian. The scenario is available to the community under GPLv3 license, and it can be downloaded from

https://github.com/lcodeca/MoSTScenario.

\section{Acknowledgements}

This work was partially funded by the French Government (National Research Agency, ANR) through the "Investments for the Future", ref. \#ANR-11-LABX-0031-01. EURECOM acknowledges the support of its industrial members, namely BMW Group, IABG, Monaco Telecom, Orange, SAP, ST Microelectronics and Symantec. 


\section{References}

[1] Jéôme Härri, Fethi Filali, and Christian Bonnet. Mobility models for vehicular ad hoc networks: a survey and taxonomy. Communications Surveys \& Tutorials, IEEE, 11(4):19-41, 2009.

[2] Francisco J Martinez, Chai Keong Toh, Juan-Carlos Cano, Carlos T Calafate, and Pietro Manzoni. A survey and comparative study of simulators for vehicular ad hoc networks (VANETs). Wireless Communications and Mobile Computing, 11(7):813-828, 2011.

[3] Daniel Krajzewicz, Jakob Erdmann, Michael Behrisch, and Laura Bieker. Recent Development and Applications of SUMO - Simulation of Urban MObility. International Journal On Advances in Systems and Measurements, 5(3\&4):128-138, December 2012.

[4] Christoph Sommer, Reinhard German, and Falko Dressler. Bidirectionally coupled network and road traffic simulation for improved IVC analysis. Mobile Computing, IEEE Transactions on, 10(1):3-15, 2011.

[5] Daniel Krajzewicz, Robbin J Blokpoel, Fabio Cartolano, Pasquale Cataldi, Ainara Gonzalez, Oscar Lazaro, Jérémie Leguay, Lan Lin, Julen Maneros, and Michele Rondinone. iTETRIS - A System for the Evaluation of Cooperative Traffic Management Solutions. In Advanced Microsystems for Automotive Applications 2010, pages 399-410. Springer, 2010.

[6] Lara Codeca and Jérôme Härri. Towards multimodal mobility simulation of C-ITS: The Monaco SUMO traffic scenario. In VNC 2017, IEEE Vehicular Networking Conference, Torino, ITALY, 112017.

[7] Sean Curtis, Andrew Best, and Dinesh Manocha. Menge: A modular framework for simulating crowd movement. Collective Dynamics, 1:1-40, 2016.

[8] Sandesh Uppoor and Marco Fiore. Large-scale urban vehicular mobility for networking research. In Vehicular Networking Conference (VNC), 2011 IEEE, pages 62-69. IEEE, 2011.

[9] Laura Bieker, Daniel Krajzewicz, AntonioPio Morra, Carlo Michelacci, and Fabio Cartolano. Traffic simulation for all: a real world traffic scenario from the city of Bologna. In SUMO2014 Modeling Mobility with Open Data. Deutsches Zentrum fur Luft- und Raumfahrt e.V. Institut fur Verkehrssystemtechnik, 2014.

[10] Luca Bedogni, Marco Gramaglia, Andrea Vesco, Marco Fiore, Jerome Harri, and Francesco Ferrero. The Bologna Ringway dataset: improving road network conversion in SUMO and validating urban mobility via navigation services. Vehicular Technology, IEEE Transactions on, 64(12):54645476, 2015.

[11] Karl-Heinz Kastner and Petru Pau. Experiences with SUMO in a Real-Life Traffic Monitoring System. In SUMO2015 - Intermodal Simulation for Intermodal Transport. Deutsches Zentrum fur Luft- und Raumfahrt e.V. Institut fur Verkehrssystemtechnik, 2015.

[12] MESO: mesoscopic version of SUMO. (Last access: March 2018).

[13] Agata Grzybek, Grégoire Danoy, and Pascal Bouvry. Generation of realistic traces for vehicular mobility simulations. In Proceedings of the second ACM international symposium on Design and analysis of intelligent vehicular networks and applications, pages 131-138. ACM, 2012.

[14] Lara Codecá, Raphaël Frank, Sébastien Faye, and Thomas Engel. Luxembourg SUMO Traffic (LuST) Scenario: Traffic Demand Evaluation. IEEE Intelligent Transportation Systems Magazine, 9(2):52-63, 2017.

[15] Mordechai Haklay. How good is volunteered geographical information? A comparative study of OpenStreetMap and Ordnance Survey datasets. Environment and planning. B, Planning E design, $37(4): 682,2010$.

[16] JOSM wiki page. https://josm.openstreetmap.de/. (Last access: March 2018).

[17] NETCONVERT wiki page. http://sumo.dlr.de/wiki/NETCONVERT. (Last access: March 2018).

[18] Michael Balmer, Kay Axhausen, and Kai Nagel. Agent-based demand-modeling framework for large-scale microsimulations. Transportation Research Record: Journal of the Transp. Res. Board, (1985):125-134, 2006. 